\title{
Estrategias de sostenibilidad ambiental de aerolíneas comerciales que contribuyen al cumplimiento de la agenda 2030
}

\author{
Nancy Patricia Caviedes Castillo \\ caviedec@poligran.edu.co \\ Politécnico Grancolombiano
}

\begin{abstract}
Resumen
Con el paso del tiempo el transporte aéreo ha tenido un crecimiento exponencial y la demanda por parte de los usuarios ha aumentado notablemente considerándolo como el medio de transporte más seguro, rápido y con mayor demanda a nivel mundial, como resultado de este crecimiento las aerolíneas han sido foco de atención debido a los contaminantes que se producen en su operación área ya que representan el $2 \%$ de emisiones de dióxido de carbono en el mundo. El presente trabajo se sustenta en la revisión documental que se enfoca en las diferentes estrategias que utilizan aerolíneas comerciales y los esfuerzos que han aunado para minimizar los impactos ambientales negativos que producen para contribuir a la sostenibilidad en la industria y aportar al cumplimiento de los Objetivos de Desarrollo Sostenible planteados por la Organización de Naciones Unidas direccionados a la protección del medio ambiente.
\end{abstract}

Palabras claves

sostenibilidad, aerolíneas, medio ambiente, pacto global, objetivos de desarrollo ambiental. 


\section{Introducción}

Actualmente, el planeta ha sufrido graves daños causados por la contaminación ambiental como consecuencia del modelo insostenible que el ser humano ha llevado a cabo, esto se evidencia en el informe "Perspectivas del medio ambiente mundial" (GEO, sus siglas en inglés) presentado por el Programa de la Naciones Unidas para el medio ambiente (PNUMA) que sustenta en un informe de 740 páginas los principales problemas ambientales del planeta y se fundamenta en el conocimiento científico a cerca de lo que está sucediendo y podría ocurrir si no se toman acciones inmediatas para mitigar el impacto ambiental.

La revista científica Environmental Research Letters (2019) afirma en su publicación "Marginal climate and air quality costs of aviation emissions" que "las emisiones de la aviación civil comercial son un contribuyente cada vez más significativo al cambio climático antropogénico", y añade que "las emisiones de dióxido de carbono (CO2) atribuibles a la aviación han aumentado un 2,6\% anual en promedio durante los últimos 25 años (Agencia Internacional de Energía 2017), y se estima que la aviación comercial ya representa aproximadamente el $5 \%$ del forzamiento global del clima". (Groble, 2019)

Los organismos reguladores de la aviación comercial como la Organización de Aviación Civil Internacional $(\mathrm{OACl})$, consciente de minimizar el impacto ambiental "estableció 15 objetivos estratégicos que están relacionados directamente con 17 objetivos de desarrollo sostenible (ODS) de las Naciones Unidas, asumiendo el compromiso de trabajar en estrecha cooperación con los Estados y con otros organismos 
de la ONU en pos de las metas comunes." (OACl, 2020). Por otro lado, la Asociación Internacional de Transporte Aéreo- IATA, en el comunicado No. 72 del 12 de diciembre de 2019 "confirma que las emisiones de carbono por pasajero han descendido más del 50\% desde 1990. La IATA atribuye este resultado en buena medida a la mejora de la eficiencia de combustible de la industria en un 2,3\% anual desde 2009 $-0,8$ puntos porcentuales más respecto al objetivo inicial—, una eficiencia que la industria ha conseguido gracias a la inversión en aviones y operaciones más eficientes." (IATA, 2019)

La importancia de la información incluida en este documento se fundamenta en adquirir conocimiento acerca de las estrategias y gestión que han desarrollado organizaciones internacionales de aviación y algunas aerolíneas comerciales para mitigar el impacto ambiental. El análisis realizado se enfoca en el área temática de Medio Ambiente que compone una de las iniciativas del Pacto Global de las Naciones Unidas junto con el reporte comparativo sobre sostenibilidad de las aerolíneas comerciales.

El objetivo de estudio de este documento es recopilar algunas estrategias de sostenibilidad ambiental implementadas por las aerolíneas comerciales de diferentes países para el cumplimiento de los Objetivos de Desarrollo Sostenible propuestos en la Agenda 2030, en la Asamblea General de las Naciones Unidas, que de acuerdo con la Resolución aprobada el 25 de septiembre de 2015 centra su interés en cinco esferas (personas, planeta, prosperidad, paz y alianzas) que son de importancia crítica para la humanidad y el planeta. (ONU, 2015). 
Para este documento se realizó una investigación explicativa que de acuerdo con Sabino (1992) se define como:

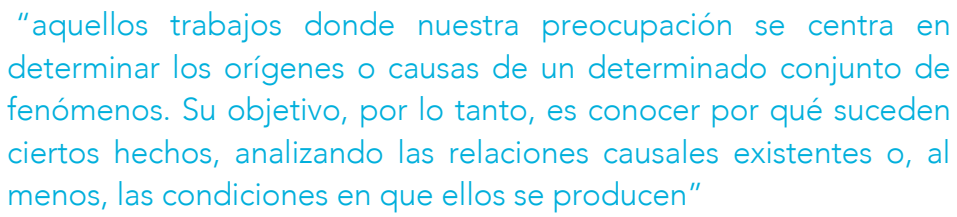

También es de tipo documental que se fundamenta en "la búsqueda de una respuesta específica a partir de la indagación en documentos. Entendamos por documento como refiere Maurice Duverger todo aquello donde ha dejado huella el hombre en su paso por el planeta" como precisa (Baena, 2014) y que se sustenta por estar enfocada específicamente en la esfera "Planeta" de la Agenda 2030 para conocer el impacto ambiental de la aviación comercial como consecuencia del crecimiento de la industria a nivel mundial; al igual que las iniciativas y actividades presentadas en los reportes de sostenibilidad de 2019 de diferentes aerolíneas que han sobresalido por establecer estrategias efectivas que ayudan a la disminución del impacto ambiental.

\section{Marco Teórico}

\section{Crecimiento de la aviación comercial a nivel mundial}

La aviación comercial es una industria global que ha experimentado un crecimiento considerable de operaciones. De acuerdo con cifras 
previas presentadas por la Organización de Aviación Civil Internacional

$(\mathrm{OACl})^{2}$

"los servicios aéreos regulares transportaron un total de 4300 millones de pasajeros en 2018, registrando un incremento del 6,1\% respecto de 2017. El número de salidas alcanzó los 38 millones en todo el mundo y el tráfico de pasajeros, expresado como total de pasajeros-kilómetros de pago (RPK) en los servicios regulares, se ubicó en los 8200 billones con un marcado aumento del 6,7\% que no alcanzó, sin embargo, a equiparar el 7,9\% logrado en 2017" (OACl, 2018).

Crecimiento en 2018 de los pasajeros-kilómetros de pago (RPK) de los servicios regulares internacionales

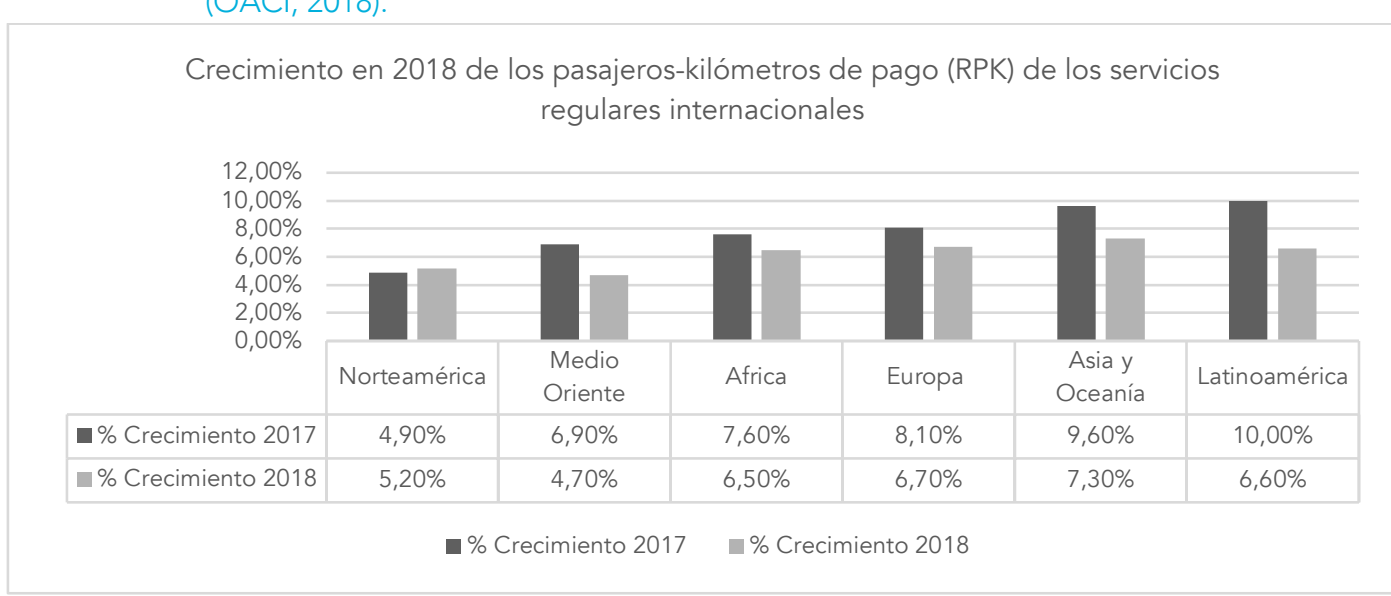

Grafica 1 Cifras preliminares difundidas por la OACl del crecimiento en 2017 y 2018 de los pasajeroskilómetros de pago (RPK) de los servicios regulares internacionales. Fuente: Elaboración propia adaptado de $(\mathrm{OACl}, 2018)$

El incremento del tráfico de pasajeros internacionales en vuelos regulares en términos de pasajeros*kilómetros de pago (RPK) para 2018 creció el 6,4\%, un descenso respecto a 2017 donde se registró un crecimiento de $8,4 \%$. En la gráfica 1 , se evidencia que todas las regiones en 2018 sufrieron un menor crecimiento comparado con el año 2017, con excepción de Norteamérica que no se vio afectada como consecuencia al afianzamiento de la economía estadounidense

2 "Es un organismo especializado de la ONU, creado por los Estados en 1944 para ejercer la administración y velar por la aplicación del Convenio sobre Aviación Civil Internacional (Convenio de Chicago)" Fuente: $(\mathrm{OACl}, 2020)$ 
y la constante expansión internacional de los transportistas canadienses. La región de Medio Oriente se posicionó en el último lugar con el 4,7\% debido a varios factores como la competencia, la baja de los precios del petróleo, la multiplicación de aeropuertos focales y las tensiones geopolíticas.

Crecimiento de la aviación comercial en Latinoamérica

El crecimiento económico de Latinoamérica es variado, en 2019 fue liderado por Panamá $(3,5 \%)$, Colombia $(3,3 \%)$ y Perú $(2,6 \%)$, de acuerdo con el Banco Mundial, sin embargo, para 2020 en su informe anual de Perspectivas Económicas Mundiales, pronostica que "la economía a nivel mundial se reducirá en un 5,2\%. En el caso de América Latina y el Caribe, el organismo estima que se registrará una caída de 7,2\% al cierre de este año" (Vargas , 2020), citó la organización en su informe, como consecuencia de la coyuntura actual sufrida por la COVID 193, sin embargo, a pesar de los desafíos actuales, la aviación comercial tiene un panorama positivo en América Latina.

3 La "COVID-19 es la enfermedad infecciosa causada por el coronavirus que se ha descubierto más recientemente. Tanto este nuevo virus como la enfermedad que provoca eran desconocidos antes de que estallara el brote en Wuhan (China) en diciembre de 2019. Actualmente la COVID-19 es una pandemia que afecta a muchos países de todo el mundo". Fuente: (Organizacion Mundial de la Salud, 2020) 


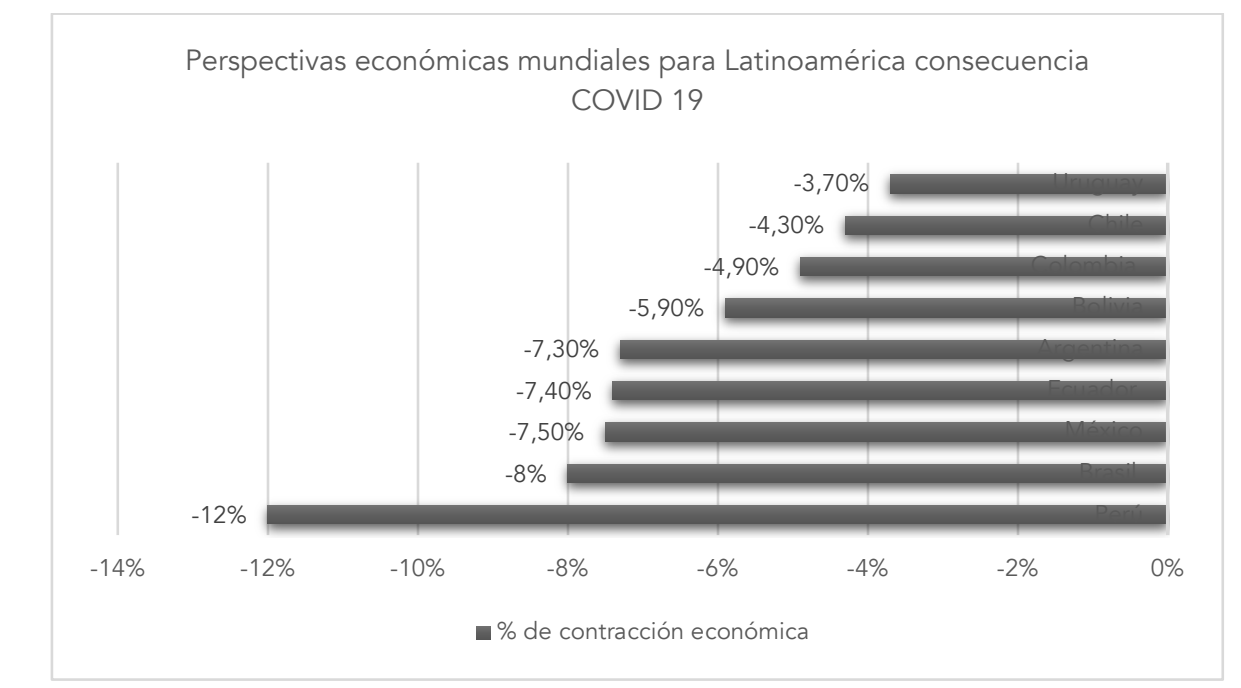

Ilustración 2 Perspectivas económicas mundiales del Banco Mundial. Fuente: Elaboración propia adaptado de https://www.bancomundial.org/es/publication/global-economic-prospects

La Asociación Latinoamericana y del Caribe de Transporte Aéreo (ALTA) informó en el mes de febrero de 2020 que las aerolíneas de la región transportaron 307.3 millones de pasajeros en 2019 lo que representó un aumento del 3.9\% comparado con el 2018, sin embargo, se pronostica que para finales de 2020 la reducción de vuelos y pasajeros transportados en la región llegaría al 49\%, consecuencia de la COVID 19, afectando el crecimiento de 16 años consecutivos donde se evidenció un aumento doble en la última década.

ALTA estima que se retomarían los niveles de antes de la crisis "a finales de 2021 (por ser una región que necesita el transporte aéreo y promisoria en estimaciones de crecimiento) o en la mitad de 2022, empezando por el mercado doméstico". (Lyonnet, 2020) 
Impactos ambientales de la aviación comercial

Las emisiones acústicas, gases y partículas producidas por los motores de las aeronaves han contribuido a la decadencia ambiental. La aviación produce el $2 \%$ de dióxido de carbono que hacen parte de los gases de efecto invernadero que aportan negativamente al calentamiento global. Por otro lado, de acuerdo con las declaraciones de Eloise Marais del Grupo de Composición Atmosférica de la Universidad de Leicester, Reino Unido argumenta que "el efecto climático de las emisiones no CO2 de la aviación es mucho mayor que el equivalente de otros medios de transporte. Estos gases de efecto invernadero no $\mathrm{CO} 2$ formados en altitudes más elevadas persisten por más tiempo que la superficie y también tienen un potencial de calentamiento más fuerte" (BBC News Mundo, 2019).

De acuerdo con (Alonso \& Ruiz, 2016), "la Commission of the European Communities, establece que los tipos de impacto ambiental del transporte aéreo se pueden clasificar como de efecto local (ruido, contaminación de aire local, uso de espacio), o de efecto global (consumo de materiales no renovables, aportación al cambio climático)".

Los impactos al medio ambiente local que afectan las zonas entorno al aeropuerto y estas se clasifican en:

- Ruido: expertos de la industria aeronáutica aseguran que la contaminación acústica es una problemática persistente que afecta la salud de pasajeros, tripulaciones y personal que trabaja en el aeropuerto. La Administración Federal de Aviación de los Estados Unidos (FAA) sustenta que la exposición continua y prolongada a los altos niveles de ruido producidos por las aeronaves pueden causar pérdida auditiva permanente. De igual manera, la continua exposición al ruido puede reducir la memoria y aumentar el cansancio en las personas, de acuerdo con estudios realizados por la Universidad de Nueva Gales del Sur. 
Los motores y el ruido aerodinámico ${ }^{4}$ son las principales fuentes generadoras, razón por la cual, la $\mathrm{OACl}^{5}$ en su anexo 16 "Protección del medio ambiente", volumen I "Ruido de aeronaves" estableció normas cuyas restricciones aumentan a medida que la tecnología avanza para contrarrestar el impacto acústico.

- Emisiones que generan contaminación del aire local: "El alcance de la industria tiene otras cifras superlativas: 1.303 líneas aéreas vuelan 31.717 aviones en 45.091 rutas entre 3.759 aeropuertos en el espacio aéreo administrado por 170 proveedores de servicios de navegación aérea" (Alonso J. , 2019). Cada una de esas aeronaves requieren para su llegada o salida atención en tierra, lo que implica uso de equipos auxiliares que emiten $\mathrm{CO} 2$, tales como, unidades generadoras de energía, aire acondicionado, tractores, paymover, conveyor, entre otros.

Al igual que el ruido, la $\mathrm{OACl}$ en el Anexo 16, Volumen II establece niveles máximos de cuatro sustancias resultantes de las emisiones producidas por la combustión del queroseno: partículas sólidas visibles (humo), hidrocarburos no quemados $(\mathrm{HC})$, monóxido de carbono (CO) y óxidos de nitrógeno (NOx).

- Uso del espacio: "La Organización de Aviación Civil Internacional (OACl) estima que para el año 2040, el número de pasajeros aéreos a nivel mundial llegaría a 10.000 millones, lo que representaría aproximadamente 90 millones de operaciones aéreas" (CEPAL, 2019), esto implica modificar y ampliar la infraestructura aeroportuaria, lo que conlleva a afectación de ecosistemas, impacto paisajístico, contaminación de agua y suelos, entre otras degradaciones ambientales.

\section{Con respecto a los impactos globales se dividen en:}

- Consumo de materiales no renovables: el combustible fósil como el queroseno utilizado en la aviación civil y militar es el resultado de una ligera fracción obtenida de la destilación del petróleo en refinería y es el componente principal del combustible de las aeronaves

\footnotetext{
4 Es el ruido producido por el flujo de aire contra el fuselaje del avión. Fuente: (Universidad de Valladolid, 2020) 5 Siglas de Organización de Aviación Civil Internacional.
} 
comerciales denominado Jet fuel ${ }^{6}$ y cuyo consumo en 2019 fue estimado por IATA en 360 millones de litros de combustible.

- Otras fuentes fósiles utilizadas en menor medida son las arenas bituminosas (una combinación de arcilla, arena, agua y bitumen), esquistos bituminosos (extracción de gas y petróleo) y el gas natural condensado, al igual que algunos metales como aluminio que al alearse con magnesio, berilio, cobre, cromo, níquel, zinc, hierro, entre otros son utilizados para la construcción de la estructura del avión y el titanio es el metal de preferencia para la fabricación de los motores.

- Contribución al cambio climático: la contaminación generada por las aeronaves es del 2\% de dióxido de carbono que sumando otros gases se eleva al 5\%, esto sin tener en cuenta la contaminación generada por otros elementos que son parte de la industria aeronáutica como aeropuertos, fabricantes de aviones, equipos de atención en tierra, entre otros.

- La aviación no solo es responsable de emisiones de CO2, también produce otros gases de efecto invernadero como el vapor de agua, metano, emisiones de nitrógeno donde se encuentra el Óxido Nitroso (N2O), Mono-óxidos de Nitrógeno (NO y NO2) y Amoníaco (NH3), también hay emisiones de partículas y otros contaminantes perjudiciales para la salud humana conocidos como HAPs (Hazardous Air Pollutants) donde se encuentran el benceno, tolueno, 1,3- butadieno, acroleína y el formaldehido (HCHO). (Alonso \& Ruiz, 2016)

\section{Objetivos de Desarrollo Sostenible - ODS y la Organización de Aviación Civil Internacional - OACl}

Actualmente millones de personas viven en la pobreza, no están garantizados todos los derechos humanos para todas las personas, más del $50 \%$ de la población mundial tiene menos derechos y oportunidades, aumentan las desigualdades sociales y crecen desmedidamente los índices de contaminación y pérdida de biodiversidad.

\footnotetext{
6 Jet fuel es el término global utilizado para los tipos de combustible líquido de diversas especificaciones que se utilizan comúnmente en aviación. Fuente: (Oiltanking - Home, 2016)

${ }^{7}$ Sigla de Asociación Internacional de Transporte Aéreo
} 
En septiembre de 2015, los líderes mundiales conscientes de esta realidad decidieron en el epicentro de la Naciones Unidas, un acuerdo internacional donde se establecieron y adoptaron un total de 17 objetivos, con 169 metas y 231 indicadores que reconocen la importancia de combatir la pobreza, intervenir en el cuidado del planeta y disminuir las desigualdades. Estas metas están proyectadas para ser alcanzadas en un periodo de 15 años, es decir, para el 2030 e involucran directamente todos los gobiernos, el sector privado y la sociedad civil.

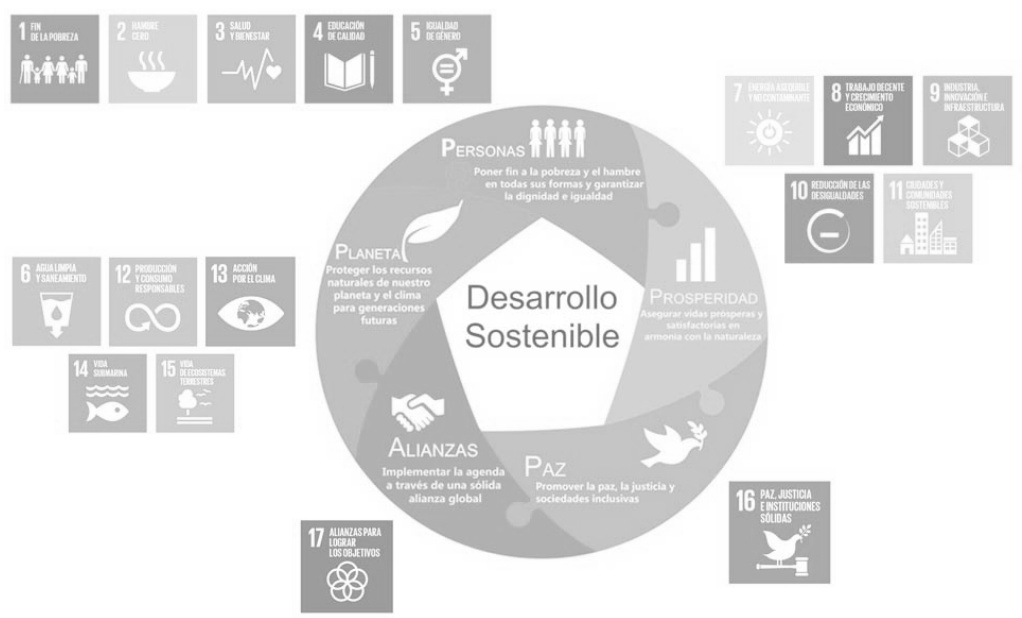

Ilustración 3 Objetivos de Desarrollo Sostenible de la Naciones Unidas. Tomado de (Programa de las Naciones Unidas para el Desarrollo, 2020)

La Organización de Aviación Civil Internacional estima que el tráfico aéreo de pasajeros y de carga aérea se duplicará en los próximo 20 años. Para 2045, el tráfico de pasajeros alcanzará más de 22 billones de RPK con un crecimiento de $4.1 \%$ anual, y el flete se expandirá en $3.6 \%$ anualmente durante el mismo período de tiempo, a 573 mil millones de FTK (toneladas por kilómetro), esto implica que se podrían aumentar las emisiones de CO2 sino se adoptan acciones y tecnología 
que ayuden a mitigar estos impactos negativos contra el medio ambiente.

La OACl es consciente que el logro de los ODS se basa en los avances en el transporte aéreo sostenible y la movilidad. Las necesidades de asistencia y capacidad de construcción, incluida la infraestructura, deben determinarse y priorizarse de conformidad con los ODS. Todas las partes interesadas deben asumir un compromiso genuino para transformar el sistema de transporte, en términos de viajes y fletes individuales, en uno que sea "seguro, asequible, accesible, eficiente y resiliente, minimizando al mismo tiempo las emisiones de carbono y otros impactos ambientales" (OACl, 2019)

\footnotetext{
"La organización se ha comprometido en adoptar todas las medidas necesarias para maximizar los beneficios de la aviación de manera sostenible, segura, eficiente, económicamente viable y ambientalmente responsable; incluyendo soluciones innovadoras y sostenibles del transporte para apresurar la implementación y apoyo de los ODS de la ONU, promoviendo la importancia de la aviación a través de una mejor conectividad aérea a nivel regional, nacional e internacional que garantice beneficios para todos". Dra. Fang Liu, Secretario General de la OACl.
}

Los objetivos estratégicos de la Organización están direccionados a "seguridad operacional, capacidad y eficiencia de la navegación aérea, seguridad de la aviación y facilitación, desarrollo económico del transporte aéreo y protección al medio ambiente" (OACl, 2020), se relacionan directamente con 15 de los 17 ODS de la ONU. Su principal compromiso es cooperar de manera cercana con los Estados y con otros organismos de la ONU. También interviene en calidad de observadora oficial en el Grupo Interinstitucional y de Expertos sobre los Indicadores de los ODS protegiendo en especial el indicador 9.1.2, "Volumen de transporte de pasajeros y carga, desglosado por medio de transporte", 
que hace parte del objetivo 9 que apunta a "Construir infraestructuras resilientes, promover la industrialización inclusiva y sostenible y fomentar la innovación" de la Agenda 2030.

En la imagen a continuación se presentan los 15 Objetivos de Desarrollo Sostenible adoptados por la OACl y los objetivos estratégicos a los cuales impactan directamente
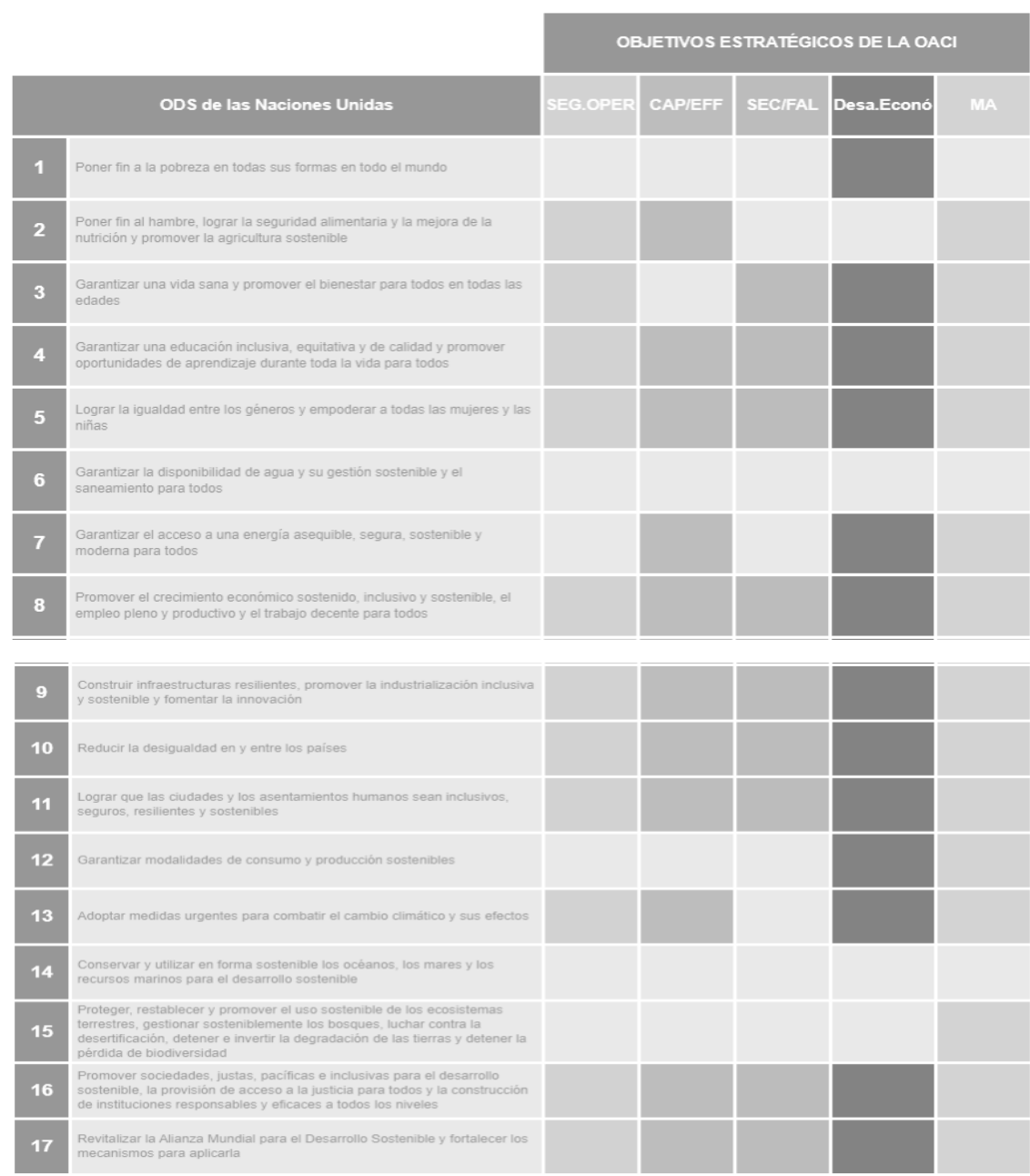

Estrategias de las aerolíneas para el desarrollo sostenible ambiental

El cambio climático es un reto que la industria aeronáutica enfrenta permanentemente como consecuencia a la contribución global del 2\% 
de las emisiones de Dióxido de Carbono, lo que equivale al 14\% de los diferentes tipos de transporte, frente al 74\% de los desplazamientos en carretera. De acuerdo con la IATA para 2037 el número de pasajeros podría ascender a 8.200 millones y para 2050 las emisiones de CO2 aumentarían a un $14 \%$.

Para contrarrestar los impactos ambientales, algunas aerolíneas comerciales han generado iniciativas para migrar a convertirse en sostenibles ambientalmente y contribuir a los Objetivos de Desarrollo Sostenible de la ONU. A continuación, se presentarán algunos ejemplos donde se recopilan de manera general cada una de las estrategias adoptadas por las aerolíneas con mayor impacto en la reducción de huella de carbono.

\section{KLM Royal Dutch Airlines}

La aerolínea holandesa KLM fue fundada el 7 de octubre de 1919, es la primera aerolínea comercial del mundo, es una de las compañías con mayor compromiso para minimizar el impacto al medio ambiente, por esta razón, lleva años liderando iniciativas con diferentes características para aumentar la sostenibilidad ambiental, y como resultado lleva más de 10 años liderando el Índice Dow Jones Sustainability (DJSI)8 que identifica a las aerolíneas más sostenibles del mundo.

8 "El Índice de Sostenibilidad Dow Jones -DJSI, por sus siglas en inglés- es un índice que desde 1999 elabora la empresa RobecoSAM, basado en el análisis de más de 600 variables, las cuales atienden a factores ambientales, sociales y de gobernanza. Se trata del primer índice mundial para identificar y clasificar a las principales empresas sostenibles". Fuente: (National Geographic, s.f.) 
Algunas de las estrategias incluidas por KLM para alcanzar el objetivo de sostenibilidad ambiental son:

- Impulsar el uso de biocombustibles que son elaborados a partir de biomasa, una materia prima de origen vegetal o animal, logrando disminuir hasta un $80 \%$ de las emisiones del avión.

- Reducir y reciclar de los plásticos de un solo uso de plásticos PET para procesarlos en filamentos para material de impresión 3D que sirven como componentes de herramientas para el mantenimiento de las aeronaves.

- Renovar su flota con los Boeing 787 Dreamliner que son aviones fabricados en su gran mayoría con fibra de carbono y otras aleaciones, convirtiéndolos en más ligeros, lo que conlleva a un $20 \%$ menos de consumo de combustible y una reducción de ruido del $40 \%$.

- Fomentar el uso del tren para distancias cortas

- Con el programa CO2ZERO es un servicio voluntario de compensación de CO2 que invita al pasajero a compensar su huella de carbono.

- Reciclaje de residuos de catering donde los tripulantes de cabina recogen de manera segregada los desechos que son separados en 12 grupos (cartón, polietileno, cristal, latas, etc.) para ser reciclados y aquellos que no pueden serlo se queman.

- En 2010 cambió los uniformes de todos los colaboradores de la aerolínea y convirtió los 90 mil kilos de tela de estos en material reciclable que fueron usados como material para las alfombras de las cabinas World Business Class de sus aeronaves.

- Desde 2014 eliminó el uso de pitillos, también conocidas como pajillas o popotes y bolsas plásticas para la venta de productos en el Duty Free a bordo.

- Con la implementación a bordo de la aplicación KLM Media logró reducir 360 mil kg de peso y desperdicio.

El Sustainability Report 2019 de AIRFRANCEKLM Group, la gestión de la aerolínea contribuyó con 6 de los 17 ODS de la ONU. En la siguiente imagen se identifican aquellos que contribuyeron positivamente con el impacto ambiental: 

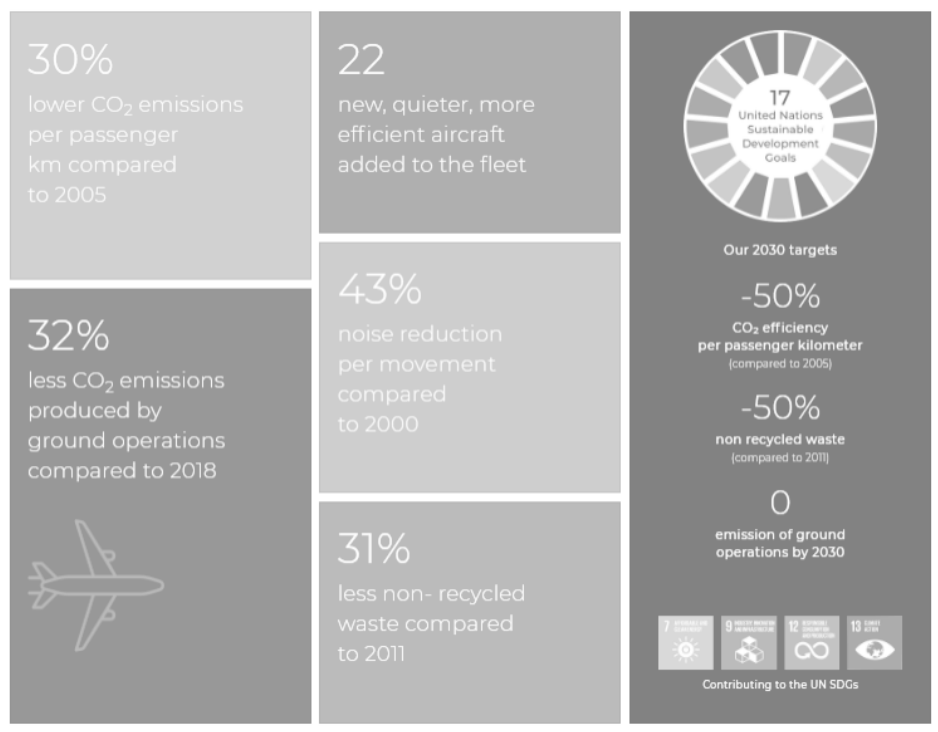

Ilustración 4 Contribución de KLM a los ODS Ambientales. Fuente: Reporte de Sostenibilidad de KLM 2019. Tomado de (AIRFRANCEKLM GROUP, 2019)

LATAM Airlines: una de las aerolíneas más sostenibles

LATAM Airlines es una aerolínea chilena que tiene sus inicios como LAN (Línea Aérea Nacional - Chile) en 1983, pero es en 2012 cuando se fusiona con la aerolínea Brasilera TAM (Transportes Aéreos Meridionáis) consolidándose como LATAM AIRLINES GROUP.

En 2019, la aerolínea se posesiona entre las tres más sostenibles a nivel mundial, de acuerdo con el Índice Dow Jones Sustainability (DJSI) gracias a sus iniciativas:

- Con su programa "Cuido mi destino" apoya el turismo sostenible con la reparación y reconstrucción de sitios turísticos, áreas verdes y fomentar las tradiciones locales con el apoyo de personas voluntarias.

- En 2017 redujo su huella de carbono en 2,46\% y con su filial de LATAM Airlines Colombia logró que sus vuelos domésticos tuvieran cero emisiones de carbono.

- En Colombia y Perú en cuanto a las operaciones en tierra alcanzó cero emisiones de carbono. 
- En 2019 LATAM Airlines Chile firmó un acuerdo para el uso de energías 100\% renovables en su edificio corporativo y base de mantenimiento.

De acuerdo con el informe de Sostenibilidad de LATAM de 2019,

"el análisis confirmó la conexión más efectiva de LATAM con siete de

los 17 objetivos de desarrollo sostenible y, en particular con el ODS

13, que trata de las acciones para combatir el cambio climático".

(LATAM Airlines, 2019)

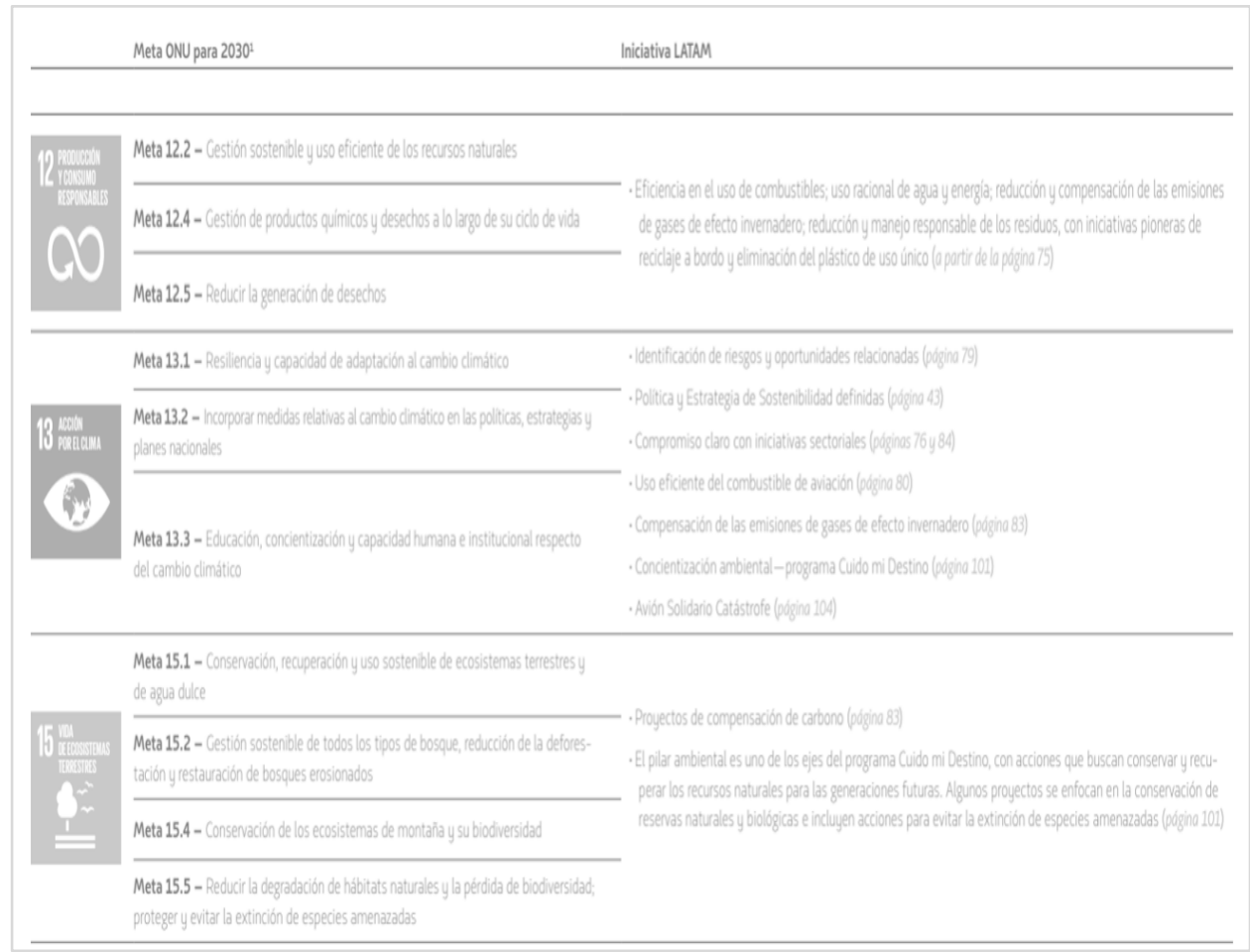

Ilustración 5 Metas e Iniciativas de LATAM Airlines para el cumplimiento de la Agenda 2030. Fuente: Reporte de sostenibilidad LATAM Airlines 2019 tomado de (LATAM Airlines, 2019)

\section{Delta Airlines}


Con una inversión proyectada de 1.000 millones de dólares la aerolínea líder en Estados Unidos, fundada el 1925, provee convertirse en una línea aérea neutral en carbono a nivel mundial. Para alcanzar este objetivo, sus iniciativas son:

- Entregó el $25 \%$ de su flota para actualizarla en los próximos 5 años.

- Uso de combustibles sostenibles.

- Limitó voluntariamente las emisiones de gases de efecto invernadero, generando una disminución de 16 millones de emisiones de $\mathrm{CO} 2$.

- En 2007, la aerolínea norteamericana fue la primera en su país en implementar un programa de reciclaje en vuelo, desde esa fecha a reciclado más de 1.500 toneladas de aluminio de los desechos abordo y complemento esta decisión en 2018 con un programa de compostaje.

- En 2018, inició el reemplazo de plásticos de un solo uso a bordo, y actualmente, cada año elimina 150 toneladas de desechos.

Delta Airlines ha aportado con 6 de los 17 Objetivos de Desarrollo Sostenible que incluyen los ODS 12 y 13 que impactan directamente al eje ambiental del Pacto Global cuyas acciones se ven reflejadas en la siguiente imagen:

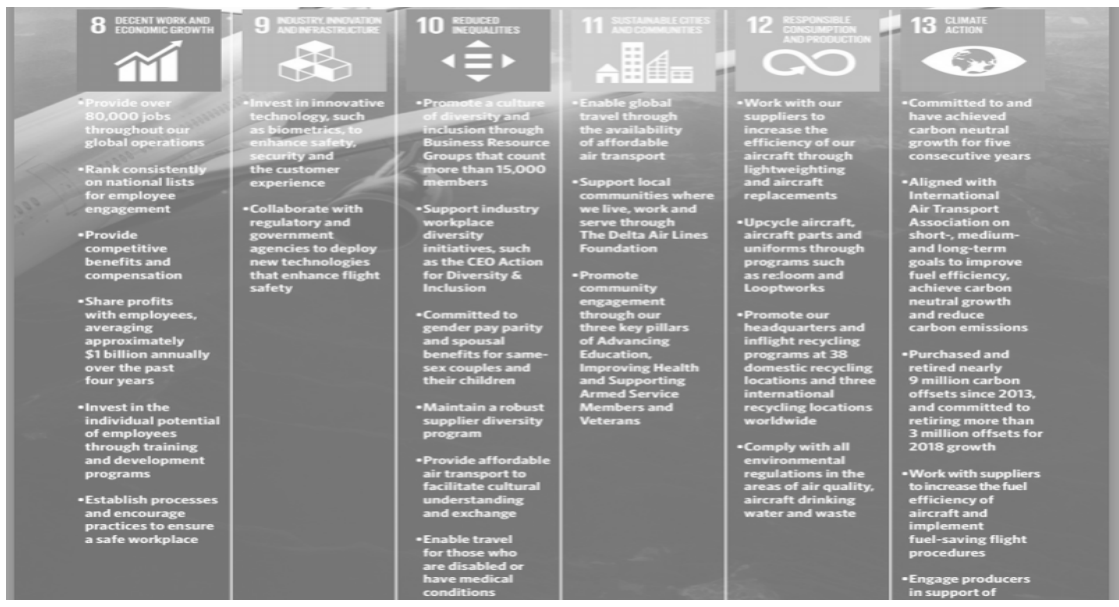

Ilustración 6 Contribución a los ODS de Delta Airlines para el cumplimiento de la Agenda 2030. Fuente: Corporate Responsibility Report 2018. Tomado de (Delta Airlines, 2018)

\section{Air France}


La aerolínea francesa fundada en 1933 hace parte del Air FranceKLM Group desde 2003, ha optimizado su modelo operativo, aumentado los ingresos de los pasajeros, aprovechado la consolidación europea, desarrollando los datos del cliente, Flying Blue, Carga y E\&M y se comprometió firmemente con la sostenibilidad mundial contribuyendo a los Objetivos de Desarrollo Sostenibles de la ONU $(5 ; 7 ; 8 ; 9 ; 12$ y 13), estableciendo políticas dirigidas a reducir el impacto ambiental (AIRFRANCEKLM Group, 2019):

- Implementar políticas de reducción de emisiones de CO2 para las operaciones aéreas y terrestres e identificar objetivos de reducción ambiciosos hasta el 2030.

- Aplicar una política para asegurar las operaciones y los procedimientos operativos y comerciales para garantizar a los clientes la continuidad del servicio, en las mejores condiciones.

- Mantener un diálogo permanente con los representantes de las asociaciones locales y residentes en los alrededores de los aeropuertos y aplicar procedimientos menos ruidosos.

- Desarrollar una política para reducir, reciclar y reciclar los residuos.

- Ofrecer a los clientes soluciones de compensación y apoyar proyectos ambientales. AIRFRANCEKLM Group presenta en la siguiente imagen los resultados obtenidos en 2018 y 2019 en relación con los indicadores claves de desempeño que mitigan el impacto ambiental: 
OBJETIVOS DE DESARROLLO SOSTENIBLE

Avances y experiencias hacia su cumplimiento

\begin{tabular}{|c|c|c|c|}
\hline \multirow{2}{*}{$\begin{array}{l}\text { Key Performance } \\
\text { Indicator } \\
\mathrm{CO}_{2} \text { efficiency } \\
\text { per passenger kilometer }\end{array}$} & \multirow{2}{*}{$\begin{array}{l}\text { Definition } \\
\text { Specific } \mathrm{CO}_{2} \text { footprint } \\
\text { for passenger transportation }\end{array}$} & \multicolumn{2}{|c|}{ Results } \\
\hline & & $\begin{array}{l}2018 \\
80 \mathrm{~g}^{-\mathrm{CO}_{2}} \\
/ \text { passenger } / \mathrm{km}\end{array}$ & $\begin{array}{l}2019 \\
79 \mathrm{~g}^{\mathrm{C}} \mathrm{CO}_{2} \\
/ \text { passenger } / \mathrm{km}\end{array}$ \\
\hline & $\begin{array}{l}\text { Percentage change in } \mathrm{CO}_{2} \text { efficiency } \\
\text { (g.co/passenger/km) between } 2005 \text { and } 2019 \\
\text { (operational measures only) }\end{array}$ & $\begin{array}{l}2019 \\
-20 \%\end{array}$ & \\
\hline & $\begin{array}{l}\text { Percentage change in } \mathrm{CO}_{2} \text { efficiency } \\
(\mathrm{g} . \mathrm{CO} / \text { /passenger/km) between } 2005 \text { and } 2019 \\
\text { (operational measures and market-based measures) }\end{array}$ & $\begin{array}{l}2019 \\
-30 \%\end{array}$ & $\begin{array}{l}2030 \text { target } \\
50 \% \text { reduction } \\
\text { compared to } 2005\end{array}$ \\
\hline $\begin{array}{l}\mathrm{CO}_{2} \text { emissions from the } \\
\text { ground operations }\end{array}$ & $\begin{array}{l}\text { Percentage change in absolute } \mathrm{CO}_{2} \text { emissions } \\
\text { from the ground operations between } 2018 \text { and } 2019\end{array}$ & $\begin{array}{l}2019 \\
-32 \%\end{array}$ & $\begin{array}{l}2030 \text { target } \\
\text { Carbon neutrality }\end{array}$ \\
\hline Non-recycled waste & $\begin{array}{l}\text { Percentage change in non-recycled waste } \\
\text { between } 2011 \text { and } 2019 .\end{array}$ & $\begin{array}{l}2019 \\
-31 \%\end{array}$ & $\begin{array}{l}2030 \text { target } \\
50 \% \text { reduction } \\
\text { compared to } 2011\end{array}$ \\
\hline Noise footprint & $\begin{array}{l}\text { Percentage of variation of global noise energy per movement } \\
\text { Global Noise Energy calculated in } \mathrm{kJ} \text { divided by the total } \\
\text { number of movements between } 2000 \text { and } 2019 \text {. }\end{array}$ & $\begin{array}{l}2018 \\
-42 \%\end{array}$ & $\begin{array}{l}2019 \\
-43 \%\end{array}$ \\
\hline
\end{tabular}

Ilustración 7 Resultados 2018 y 2019 de AIRFRANCEKLM Group con respecto a los Indicadores claves de desempeño para mitigar el impacto ambiental. Tomado de (AIRFRANCEKLM Group, 2019)

\section{Qantas Airlines Limited}

La aerolínea bandera de Australia fue fundada en 1920 y es la tercera aerolínea comercial más antigua después de la holandesa KLM y la colombiana Avianca respectivamente. Conscientes del arduo trabajo que se debe realizar para minimizar el impacto en el medio ambiente ha liderado iniciativas de gestión del riesgo climático, reducción de emisiones, compensación de carbono, La percepción de los consumidores sobre la sostenibilidad, reducción de residuos, energía y aguas, así como, protección contra la contaminación.

\footnotetext{
"Qantas reconoce que el cambio climático inducido por el hombre es un problema importante para la industria de la aviación. La industria ha estado en la primera línea de la respuesta empresarial mundial al cambio climático, convirtiéndose en la primera industria en comprometerse voluntariamente con los objetivos de emisiones hasta el año 2050" (Qantas Group, 2020).
}

Las estrategias implementadas por la aerolínea son:

- Eficiencia de combustible mediante la modernización de la flota y mejoras operativas. El cambio se producirá en el año fiscal 21 mediante la introducción del B787-9s y la retirada del 
B747s para lograr el ,5\% de mejora media anual de la eficiencia de combustible de 2009 a 2020.

- Utilización de combustibles de aviación sostenibles y compensaciones de carbono cuando sea posible para limitar las emisiones a los niveles de 2020.

- Inversión continua en nueva tecnología aeronáutica, combustibles de aviación sostenibles y desarrollo de la industria del mercado del carbono para lograr cero emisiones para 2050

- Mejoras operativas e inversión en nuevas tecnologías que contribuyan Reducción del 35\% del consumo de electricidad para 2020 respecto a 2009, reducción del 20\% en el consumo de agua para 2020 con base en 2009 y 75\% de reducción en residuos para finales de 2021 con base a 2018.

A través de diferentes proyectos, Qantas contribuye significativamente a los Objetivos de Desarrollo Sostenible; por ejemplo, Qantas' Future Planet, es una iniciativa de sostenibilidad que surge en 2007 y que tiene como objetivo involucrar a organizaciones con ideas afines que buscan reducir su impacto ambiental y demostrar su visión a través de la innovación y la acción colaborativa, cooperando con 8 ODS.
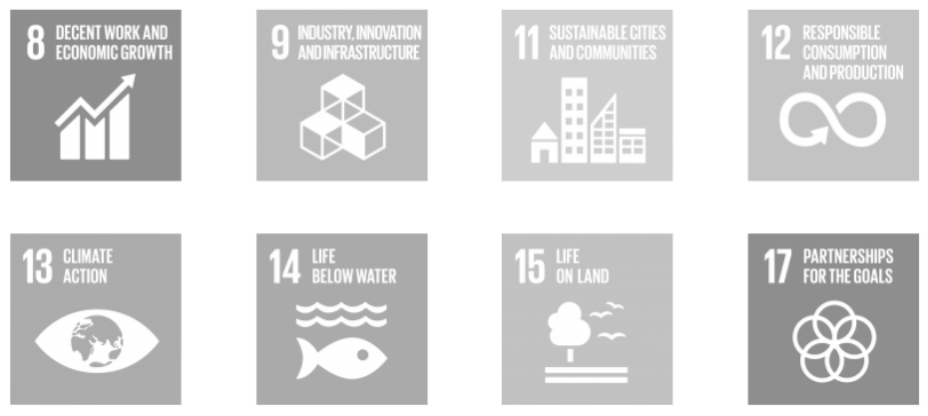

Ilustración 8 Objetivos de Desarrollo Sostenibles impactados por la iniciativa Qantas' Future Planet. Tomado de (Qantas, 2020)

A través de la inversión de Qantas en proyectos de compensación de carbono en toda Australia, el programa Qantas' Future Planet está ayudando a obtener importantes resultados sociales y medioambientales. Esto incluye el apoyo al empleo de guardas 
forestales indígenas en el norte de Australia que utilizan prácticas tradicionales para promover la regeneración de la vegetación nativa. (Qantas Group, 2020)

Iberia

Fundada en 1927, la aerolínea insignia de España está comprometida con el cumplimiento de los Objetivos de Desarrollo Sostenible (ODS) de Naciones Unidas y su principal objetivo es lograr emisiones netas cero de CO2 para 2050.

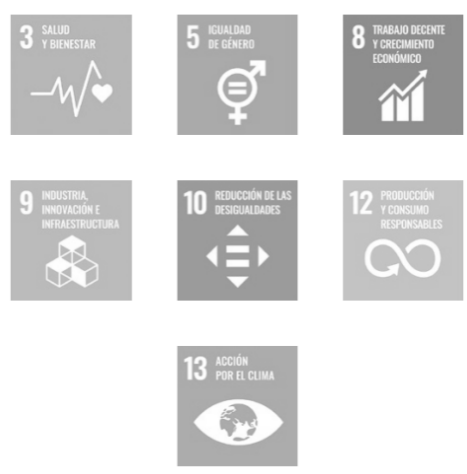

Ilustración 9 Objetivos de Desarrollo Sostenible impactados por Iberia Group. Fuente: Informe de Sostenibilidad 2019 Tomado de (Grupo Iberia, 2019)

Para contribuir a la sostenibilidad ambiental, Iberia ha desarrollo proyectos amigables con el medio ambiente:

- Estrategia Flightpath Net Zero que plantea la reducción de un 10\% de emisiones por pasajero para el 2025, aumentado a una $20 \%$ en 2025 y lograr emisiones netas cero en 2050.

- La renovación de su flota con aeronaves de última generación, más eficientes silenciosos y con avances tecnológicos significativos han reducido las emisiones de CO2 hasta un 35\% y las emisiones de oxígenos de nitrógeno en un 50\%.

- La reducción de combustible ha evitado la emisión de más de 40 mil toneladas de CO2 en 2019. 
- Con la separación a bordo de residuos, la aerolínea ha conseguido reciclar 2.253 toneladas de basura, suponiendo un $42 \%$ de incremento con respecto a las acciones previas al proyecto.

- La eliminación de bolsas plásticas para envolver cobijas, endredones, auriculares, kit infantil y removedores para bebidas contribuyo a reducir 68.5 toneladas de residuos en 2019.

- Digitalizar el contenido de revistas y prensa a bordo permitió la reducción de más de 20 toneladas mensuales de residuo de papel.

- Reducción significativa de emisiones y consumo de combustible fósil como consecuencia de la renovación del $80 \%$ de los equipos para atención en tierra de las aeronaves.

- Energía eléctrica procedente de fuentes $100 \%$ renovables para las instalaciones propiedad de Iberia.

- Uso de luces led para los hangares que permitió el ahorro de energía en 1.5 millones de KWh.

- Mejora en la calidad del aire local y del ruido cumpliendo en un alto porcentaje con las normas establecidas por Comité de Protección Ambiental de la Aviación (CAEP)

\section{Avianca Holdings S.A.}

La segunda aerolínea más antigua del mundo es de origen colombiano y fue fundada en 1919 con el nombre de Sociedad Colombo Alemana de Transporte Aéreo - SCADTA, posteriormente, se fusiona en 1940 con la empresa de Servicio Aéreo Colombiano (SACO) cambiando su nombre Aerovías Nacionales de Colombia S.A - AVIANCA y en 2003 la compañía renueva su razón social por Aerovías del Continente Americano S.A.-AVIANCA y en 2013 adopta su nombre legal actual como Avianca Holdings S.A.

La aerolínea bandera de Colombia en 2019 logró ser reconocida como la quinta aerolínea más sostenible del mundo de acuerdo con el Índice de Sostenibilidad Dow Jones- MILA Pacific Alliance y con la continua implementación de actividades e iniciativas orientadas a impactar en los Objetivos de Desarrollo Sostenible para el mismo año contribuyó a 12 ODS. 
En cuanto al eje ambiental planteado en la Agenda 2030, la aerolínea ha establecido tres pilares dentro de su plan estratégico con proyección para 2020, metas que impactan a 6 objetivos para minimizar el impacto ambiental como presentan en el siguiente cuadro que indica las metas que la aerolínea desea alcanzar para dar cumplimiento a su compromiso de responsabilidad ambiental, donde se puede evidenciar que las estrategias climáticas son su prioridad.

Tabla 11 Acciones de Avianca Holdings S.A. para contribuir a la reducción del impacto ambiental conforme con lo ODS. Fuente: Elaboración propia adaptado de (Avianca Holdings S.A, 2019)

\begin{tabular}{|c|c|}
\hline \multicolumn{2}{|r|}{ Ecoeficiencia operacional } \\
\hline ODS 6 & $\begin{array}{l}\text { Meta 6.4. De aquí a 2030, aumentar considerablemente el uso eficiente de los recursos hídricos en } \\
\text { todos los sectores y asegurar la sostenibilidad de la extracción y el abastecimiento de agua dulce para } \\
\text { hacer frente a la escasez de agua y reducir considerablemente el número de personas que sufren falta } \\
\text { de agua. }\end{array}$ \\
\hline ODS 12 & $\begin{array}{c}\text { Meta } 12.5 \text { De aquí a 2030, reducir considerablemente la generación de desechos mediante actividades } \\
\text { de prevención, reducción, reciclado y reutilización. }\end{array}$ \\
\hline \multicolumn{2}{|r|}{ Estrategia climática } \\
\hline ODS 7 & Meta 7.3 De aquí a 2030, duplicar la tasa mundial de mejora de la eficiencia energética. \\
\hline ODS 12 & $\begin{array}{c}\text { Meta } 12.4 \text { De aquí a 2020, lograr la gestión ecológicamente racional de los productos químicos y de } \\
\text { todos los desechos a lo largo de su ciclo de vida, de conformidad con los marcos internacionales } \\
\text { convenidos, y reducir significativamente su liberación a la atmósfera, el agua y el suelo a fin de } \\
\text { minimizar sus efectos adversos en la salud humana y el medio ambiente. }\end{array}$ \\
\hline ODS 13 & $\begin{array}{c}\text { Meta } 13.1 \text { Fortalecer la resiliencia y la capacidad de adaptación a los riesgos relacionados con el clima } \\
\text { y los desastres naturales en todos los países. Meta } 13.3 \text { Mejorar la educación, la sensibilización y la } \\
\text { capacidad humana e institucional respecto de la mitigación del cambio climático, la adaptación a él, la } \\
\text { reducción de sus efectos y la alerta temprana. } \\
\text { Meta 13.a Cumplir el compromiso de los países desarrollados que son partes en la Convención Marco } \\
\text { de las Naciones Unidas sobre el Cambio Climático de lograr para el año } 2020 \text { el objetivo de movilizar } \\
\text { conjuntamente } 100.000 \text { millones de dólares anuales procedentes de todas las fuentes a fin de atender } \\
\text { las necesidades de los países en desarrollo respecto de la adopción de medidas concretas de } \\
\text { mitigación y la transparencia de su aplicación, y poner en pleno funcionamiento el Fondo Verde para el } \\
\text { Clima capitalizándolo lo antes posible. } \\
\text { Meta 13.b Promover mecanismos para aumentar la capacidad para la planificación y gestión eficaces en } \\
\text { relación con el cambio climático en los países menos adelantados y los pequeños Estados insulares en } \\
\text { desarrollo, haciendo particular hincapié en las mujeres, los jóvenes y las comunidades locales y } \\
\text { marginadas }\end{array}$ \\
\hline \multicolumn{2}{|r|}{ Política y sistema de gestión ambiental } \\
\hline ODS 12 & 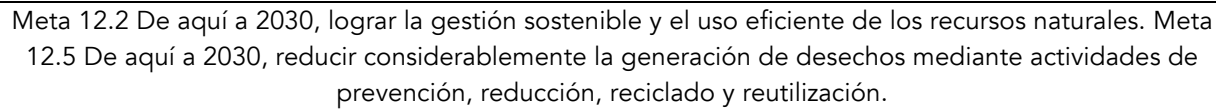 \\
\hline
\end{tabular}

Finalmente, se evidencia que el sector aeronáutico es consciente de la huella de carbono que esta produciendo y que algunas aerolíneas han desarrollado diferentes estrategias que conllevan a la mitigación del 
impacto ambiental, sin embargo, aún falta que muchas aerolíneas se unan a estos esfuerzos y generen iniciativas ambientalmente sostenibles.

\section{Conclusiones}

- El impacto ambiental producido por la industria aeronáutica se ha convertido en una situación crítica que ha captado la atención de diferentes organizaciones internacionales de aviación y de países alrededor del mundo que han desplegado iniciativas y estrategias dirigidas a enfrentar retos para acabar con la extrema pobreza, garantizar que todos los niños reciban una buena educación, lograr oportunidades iguales para todos y promover mejores prácticas de consumo y producción que ayuden al planeta ha ser más limpio y saludable para el año 2030, contribuyendo continuamente a los Objetivos de Desarrollo Sostenible propuestos por la Organización de Naciones Unidas.

- El desarrollo y crecimiento de la aviación están determinadas no solo por la demanda de pasajeros sino también por todas las acciones implementadas que favorezcan la reducción de emisiones de gases de efecto invernadero al igual que los niveles de ruido.

- Las nuevas tecnologías tienen un papel importante para lograr avances significativos en la reducción de la huella de carbono, pero por sí solas no son la solución al problema, también se deben compensar con políticas ambientales que sean el motor hacia la meta de sostenibilidad ambiental propuesta por los Organismos Internacionales y Gubernamentales de los diferentes Estados.

- El sector del transporte es responsable de $14 \%$ de emisiones de gases contaminantes, incluyendo el transporte aéreo que aporta un $2 \%$ de $\mathrm{CO} 2$ a la estadística, sin embargo, existen distintos sectores que afectan de manera contundente al medio ambiente, entre ellos el sector energético (25\%), sector industrial (21\%) seguido de otros sectores en un 16\%, de acuerdo con el Grupo Intergubernamental de Expertos sobre el Cambio Climático (IPCC por sus siglas en inglés) de las Naciones Unidas, es por esta razón, vale la pena resaltar que aunque la industria aérea sea responsable de un porcentaje mínimo comparado con los demás, esta aunando esfuerzos importantes para generar consciencia ambiental y ayudar al futuro del planeta.

\section{Referencias}

AIRFRANCEKLM GROUP. (2019). Sustainability Report 2019. Obtenido de https://sustainabilityreport2019.airfranceklm.com/

AIRFRANCEKLM Group. (2019). Universal Registration Documen. Obtenido de Reporting of the Group's extra - financial risks: 
https://www.airfranceklm.com/en/system/files/universalregistrationdocument2019va.p df

AIRFRANCEKLM Group. (2019). Universal registration Document 2019. Obtenido de Environmental Impact: https://www.airfranceklm.com/sites/default/files/universalregistrationdocument2019_2 0-04-20air_france_klm_1.pdf

Alonso, G., \& Ruiz, A. (21 de Abril de 2016). Universidad Politécnica de Madrid. Obtenido de EL IMPACTO AMBIENTAL DEL TRANSPORTE AÉREO Y LAS MEDIDAS PARA MITIGARLO: http://oa.upm.es/20345/1/INVE_MEM_2012_133532.pdf

Alonso, J. (2019). ABC Ciencia. Obtenido de Cuántos vuelos recorren cada día los cielos del mundo: https://www.abc.es/viajar/noticias/abci-cuantos-vuelos-recorren-cada-cielosmundo-201905040107_noticia.html

Avianca Holdings S.A. (2019). Informe anual 2019. Obtenido de http://s22.q4cdn.com/896295308/files/doc_financials/2019/ar/Avianca-AVH2019Abril-21-baja.pdf

Baena, G. (2014). Editorial Patria. Obtenido de Metodología de la Investigación: https://editorialpatria.com.mx/mobile/pdffiles/9786074384093.pdf

Banco Mundial Internacional. (Junio de 2020). Perspectivas Económicas Mundiales. Obtenido de https://www.bancomundial.org/es/publication/global-economic-prospects

BBC News. (24 de agosto de 2019). Science \& Environment. Obtenido de Climate change: Should you fly, drive or take the train?: https://www.bbc.com/news/scienceenvironment-49349566

BBC News Mundo. (2019). Cambio climático: ¿cuál es el medio de transporte que más contamina? Obtenido de https://www.bbc.com/mundo/noticias-49461967

CEPAL. (2019). Boletín 370 . Obtenido de Infraestructura aeroportuaria: https://repositorio.cepal.org/bitstream/handle/11362/44900/1/S1900358_es.pdf

Delta Airlines. (2018). Corporate Responsibility Report 2018. Obtenido de http://www.corporatereport.com/delta/2018/crr/Delta_2018_CRR.pdf

González Sánchez, J. L. (2010). Dialnet. Obtenido de Jugabilidad. Caracterización de la experiencia del jugador en videojuegos: https://dialnet.unirioja.es/servlet/tesis?codigo=20914

Groble, C. (2019). Environmental Research Letters. Obtenido de Marginal climate and air quality costs of aviation emissions: https://iopscience.iop.org/article/10.1088/17489326/ab4942/pdf

Grupo Iberia. (2019). Informe Sostenibilidad 2019. Obtenido de https://grupo.iberia.com/contents/archives/475/109/pdfcontent/475_109_159129730 7.pdf

IATA. (2019). Publicaciones. Obtenido de Las emisiones de carbono por pasajero descienden más del $50 \%$ 1990: https://www.iata.org/contentassets/25e5377cf53c4e48bbaa49d252f3ab03/2019-1212-01-sp.pdf

IATA. (Marzo de 2020). CANAERO. Obtenido de Indicadores de la aviaicón internacvional por Región: https://canaero.org.mx/estadistica-mundial/indicadores-de-la-aviacion- 
internacional-por-region/

LASA. (2020). Equipos de Tierra. Obtenido de http://www.lasa.com.co/index.php?option=com_content\&view=article\&id=81\&ltemid $=248$

LATAM Airlines. (2019). Memoria Integrada. Obtenido de http://www.latamairlinesgroup.net/static-files/b90aa28c-2a25-4c4f-b467cf790795908d

LATAM Airlines. (2019). Memoria Integrada 2019. Obtenido de Sostenibilidad: http://www.latamairlinesgroup.net/static-files/b90aa28c-2a25-4c4f-b467cf790795908d

Lyonnet, J. (2020). Hosteltur. Obtenido de Se pierde el crecimiento de 9 años para las aerolíneas de Latinoamérica: https://www.hosteltur.com/lat/136089_se-pierde-elcrecimiento-de-9-anos-para-las-aerolineas-de-latinoamerica.html

National Geographic. (s.f.). Sostenibilidad. Obtenido de ¿Qué es el Índice de Sostenibilidad Dow Jones?: https://www.nationalgeographic.com.es/naturaleza/actualidad/que-esindice-sostenibilidad-dow-jones_13223

OACI . (2019). Obtenido de La OACl apoya los esfuerzos de toda la ONU para reorientar la atención mundial hacia los Objetivos de Desarrollo Sostenible de la Agenda 2030 : https://www.icao.int/Newsroom/Pages/ES/ICAO-supports-UN-wide-effort-to-refocusglobal-attention-on-Agenda-2030-Sustainable-Development-Goals.aspx

OACl. (2018). Comunicados de prensa. Obtenido de Crecimiento sostenido del tráfico de pasajeros y demanda moderada de servicios de carga aérea en 2018: https://www.icao.int/Newsroom/Pages/ES/Solid-passenger-traffic-growth-andmoderate-air-cargo-demand-in-2018.aspx

OACl. (2018). Comunicados de Prensa. Obtenido de Crecimiento sostenido del tráfico de pasajeros y demanda moderada de servicios de carga aérea en 2018: https://www.icao.int/Newsroom/Pages/ES/Solid-passenger-traffic-growth-andmoderate-air-cargo-demand-in-2018.aspx

OACI. (2019). Aviation Benefits Report. Obtenido de Sustainable Air Transport Development: https://www.icao.int/sustainability/Documents/AVIATION-BENEFITS-2019-web.pdf

OACl. (2020). El Consejo de la OACl. Obtenido de Objetivos estratégicos : https://www.icao.int/about-icao/Council/Pages/ES/Strategic-Objectives.aspx

OACl. (2020). Desarrollo de la aviación. Obtenido de La OACl y los Objetivos de desarrollo sostenible de las Naciones Unidas: La OACl y los Objetivos de desarrollo sostenible de las Naciones Unidas

OACl. (2020). OACl . Obtenido de Sobre la OACl : https://www.icao.int/abouticao/Pages/ES/default_ES.aspx

OACl. (2020). Orgaización de aviaición Civil Internacional. Obtenido de La OACl y los Objetivos de desarrollo sostenible de las Naciones Unidas : https://www.icao.int/abouticao/aviation-development/Pages/ES/SDG_ES.aspx

Oiltanking - Home. (Diciembre de 2016). Glosario. Obtenido de Jet fuel : https://www.oiltanking.com/es/publicaciones/glosario/detalles/term/jet-fuel-alemankerosin.html 
ONU. (2015). Asamblea General. Obtenido de Resolución aprobada por la Asamblea General el 25 de septiembre de 2015 : https://www.un.org/ga/search/view_doc.asp?symbol=A/RES/70/1\&Lang=S

Organización de Naciones Unidas . (2015). Objetivos de Desarrollo Sostenible. Obtenido de https://www.un.org/sustainabledevelopment/es/objetivos-de-desarrollo-sostenible/

Organizacion Mundial de la Salud. (2020). Preguntas y respuestas sobre la enfermedad por coronavirus (COVID-19). Obtenido de https://www.who.int/es/emergencies/diseases/novel-coronavirus-2019/advice-forpublic/q-a-coronaviruses

Programa de las Naciones Unidas para el Desarrollo. (2020). Objetivos de Desarrollo Sostenible. Obtenido de http://americalatinagenera.org/newsite/index.php/es/laagenda-post-2015

Qantas. (2020). Qantas Future planet program. Obtenido de https://sdgs.org.au/project/qantas-future-planet-program/

Qantas Group. (2020). Our planet. Obtenido de Our targets: https://www.qantas.com/au/en/qantas-group/acting-responsibly/ourplanet.html?int_cam=au\%3Aacting-responsibly\%3Aarticle\%3Aourplanet\%3Aen\%3Ann\#our-initiatives

Qantas Group. (2020). The Australian SDG. Obtenido de Qantas' Future Planet : https://sdgs.org.au/project/qantas-future-planet-program/

Ramos, P. (11 de enero de 2019). El Tiempo. Obtenido de ¿Qué medio de transporte contamina más?: https://noticias.eltiempo.es/que-medio-de-transporte-contaminamas/

Revista Dinero. (13 de Julio de 2018). Turismo. Obtenido de Turismo: la prometedora industria que no contamina: https://www.dinero.com/pais/articulo/balance-del-sectorturismo-en-colombia-2018/260070

Sabino, C. (1992). El proceso de investigación. Obtenido de https://metodoinvestigacion.files.wordpress.com/2008/02/el-proceso-deinvestigacion_carlos-sabino.pdf

Universidad de Valladolid. (2020). Aviones. Obtenido de Ruido de aviones: https://www.lpi.tel.uva.es/ nacho/docencia/ing_ond_1/trabajos_05_06/io6/public_ht $\mathrm{ml} /$ aviones.html

Vargas, P. A. (2020). La República. Obtenido de La economía mundial se contraerá 5,2\% y la de la región 7,2\% al cierre de este año: https://www.larepublica.co/globoeconomia/laeconomia-mundial-se-contraera-52-y-la-de-la-region-72-al-cierre-de-este-ano3015797 